\title{
Palladium-Catalyzed Isomerization/(Cyclo)carbonylation of Pentenamides: a Mechanistic Study of the Chemo- and Regioselectivity
}

\author{
Bart Limburg, ${ }^{\ddagger}$ Yann Gloaguen, ${ }^{\ddagger}$ Hans M. de Bruijn, Eite Drent, and Elisabeth Bouwman* \\ Leiden Institute of Chemistry, Gorlaeus Laboratories, Leiden University, P.O. Box 9502, 2300 RA Leiden (The Netherlands) \\ $\ddagger$ Equal contributions \\ * Corresponding author; bouwman@chem.leidenuniv.nl
}

\begin{abstract}
A new isomerizing ring-closing amidocarbonylation reaction is reported using Pd-catalysis with bulky diphosphane ligands. From terminal as well as internal pentenamide isomers (PAs), cyclic imides were obtained in good yield (92\%) with cationic Pd catalysts supported by bis-PCg ligands (PCg = 6-phospha-2,4,8 trioxa1,3,5,7-tetramethyladamant-6-yl). An excess of strong acid is required to obtain high selectivity for imide products. From a low-temperature NMR study it was deduced that $\mathrm{N}$-coordination of the amide moiety is responsible for a high selectivity to cyclic imide products. In weakly acidic conditions O-coordination of the amide functionality leads to the formation of cyanoacids (i.e., 5-cyanovaleric acid, 2-methyl-4-cyanobutyric acid and 2-ethyl-3-cyanopropionic acid). It is proposed that the formation of these cyanoacids occurs through a novel intramolecular tandem de-hydrating hydroxycarbonylation reaction of PA's. This reaction also occurs in intermolecular versions of amidocarbonylation with mixtures of alkene and amide substrates. Experiments with $\mathrm{N}$-alkylated amides have been instrumental in developing mechanistic models. The strong acid co-catalyst ensures double-bond isomerization to occur faster than product formation, resulting in the same product mixture, irrespective of the use of terminal or internal pentenamides. The remaining challenge is to arrive at the desired adipimide by overcoming the undesirable regioselectivity caused by chelation of the amide.
\end{abstract}

Keywords: caprolactam $\bullet$ biomass $\bullet$ palladium-diphosphane $\bullet$ tandem isomerization/amido-carbonylation $\bullet$ imide $\bullet$ cyanoacid

\section{Introduction}

Developing alternative routes to bulk and fine chemicals based on renewable biomass is a highly desirable goal of chemical industry. Lignocellulosic biomass is the most abundantly available raw material on earth and has attracted a lot of interest as sustainable feedstock. ${ }^{[1-2]}$ Its potential conversion into various platform chemicals such as 5-hydroxymethylfurfural, ${ }^{[3]}$ furfural, levulinic acid, ${ }^{[4]}$ and $\gamma$-valerolactone $(G V L)^{[5]}$ is expected to pave the way for economical and sustainable production of various bulk chemicals and liquid fuels. For these compounds a sufficient volume of operation can be achieved to significantly impact the chemical industry's ambitions to reduce greenhouse gas emissions. $\varepsilon$-Caprolactam (CAP) is an example of a potentially accessible bulk chemical from biomass; CAP serves as the precursor for nylon-6, an extensively produced polymer (about 4 Mt/year). CAP's traditional synthesis from oil-based feedstock involves high energy consumption and substantial salt coproduction. ${ }^{[6-7]}$

We have shown that bio-based GVL can be converted into a mixture of isomeric pentenamides (PAs), which ultimately yields $\varepsilon$-caprolactam via a Rh-catalyzed tandem isomerizing intramolecular hydroamidomethylation reaction i.e. isomerization, hydroformylation, cyclization and subsequent hydrogenation (Scheme 1 , right). ${ }^{[8-9]}$ The major limitation for the viability of this overall process, in terms of both activity and selectivity, is the rather poor performance of the Rh/Xantphos catalyst for the selective transformation of the internal olefins 2-PA and 3-PA, the latter being the major isomer obtained from GVL. The rate of isomerization was shown to be lower than the rate of hydrogenation, resulting in substantial pentanamide (valeramide) production and thus severe 
feedstock loss. For a realistic process based on GVL and using this Rh-catalyst, an energy-intensive preisomerization/separation step of the mixture of PAs would be required.

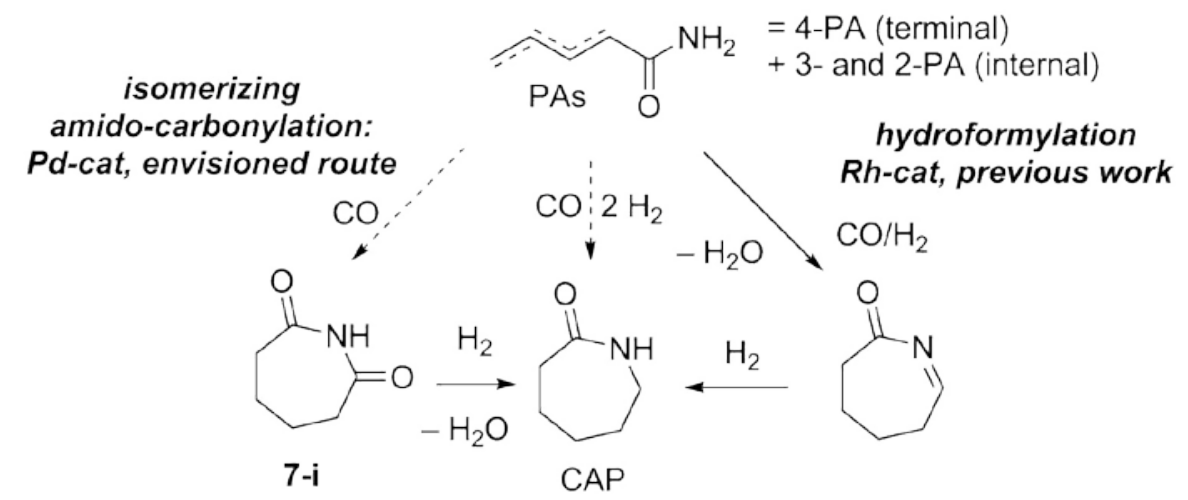

Scheme 1. Previously reported (Rh-cat, right) ${ }^{[8-9]}$ and envisioned catalytic route (Pd-cat, left) for the conversion of (biomass-derived) pentenamides to caprolactam.

In recent years, palladium catalysts have found widespread use in alkene carbonylation reactions such as hydroxy/alkoxy- as well as amino- ${ }^{[10-15]}$ and amido-carbonylation processes. ${ }^{[16]}$ Importantly, $\mathrm{Pd}(\mathrm{II})$ catalysts bearing electron-rich, bulky diphosphane ligands (typically bis(di-tert-butylphosphanyl)xylene, L1), have been shown to efficiently promote the selective isomerizing hydroxy/methoxy carbonylation of internal alkenes endowed with a functional group (acid or ester) to linear di-acids/esters. ${ }^{[17-19]}$ The high regioselectivity for linear carbonylation products with these catalysts can be seen as a consequence of the almost exclusive carbonylation at the terminal $\mathrm{C}$-atom of a paraffinic chain. This unique attribute can, in turn, be ascribed to two underlying catalytic phenomena: (i) rapid 'running' of $\mathrm{Pd}-\mathrm{H}$ species, under a $\mathrm{CO}$ atmosphere, along the carbon chain followed by (ii) an overall rate-determining termination reaction (forming e.g. acid or ester product) from the $\mathrm{Pd}$-acyl species with regeneration of the starting Pd-H species. The latter occurs via attack of the nucleophilic reagent (alcohol/water) at the Pd-acyl center, which is mainly governed by steric factors induced by the supporting bulky ligand. As a consequence, termination will proceed much faster at the least sterically hindered terminal C-atom, thus producing a linear carboxylic acid or ester with high selectivity.

Inspired by this chemistry, we took up the challenge to develop an intramolecular isomerizing carbonylation reaction, applied to the substrate PA and aiming to form selectively adipimide 7-i (Scheme 1, left). ${ }^{[20-21]}$ We thus envisioned another route to (bio-based) CAP that separates the isomerizing carbonylation of PA from reduction by $\mathrm{H}_{2}$. In this way, loss of PA by hydrogenation will be prevented, whereas the same high overall theoretical atom-efficiency in CAP production from PA can be achieved. ${ }^{[22-23]}$ Semi-hydrogenation of lactams has indeed been reported previously. ${ }^{[22]}$ Herein, we present the results of our investigations into the intramolecular amidocarbonylation of pentenamides. 


\section{Results}

\section{Pd-catalyzed amidocarbonylation of pentenamides}

Initially, the $\mathrm{Pd}$ "/L1/methane sulfonic acid (HOMs) catalyst system reported by Low et al. ${ }^{[19]}$ was applied to the substrate 4-pentenamide (4-PA). A disappointingly low yield of cyclic imide carbonylation products (5-i/6-i) was obtained (Table 1, entry 1 ). Both an in situ mixture of $\mathrm{Pd}(\mathrm{OAC})_{2} / \mathrm{L} 1 / \mathrm{HOMs}$ or a mixture of the preformed complex $[\mathrm{Pd}(\mathbf{L} \mathbf{1})(\mathrm{dba})]$ and HOMs were used as catalyst precursors; both procedures gave essentially the same results (Table 1, entry 1 vs. entry 2). The yield of any of the possible cyclo-amidocarbonylation products that can be produced in this carbonylation reaction appears to be rather low using ligand L1; in particular the desired seven-membered cyclic imide product 7-i corresponding to cyclo-carbonylation at the terminal carbon atom was not observed.

After surveying a series of parameters (type of ligand, type of Pd source, type of acid, type of solvent and CO pressure, Tables S2-6) in the carbonylation of PA isomers, we identified catalytic systems and conditions for formation of intramolecular carbonylation products $\mathbf{5 - i}$ and $\mathbf{6}-\mathbf{i}$ in good yields. The bulky "cage ligands" $\mathbf{L} \mathbf{2}$ and L3 (PCg = 6-phospha-2,4,8-trioxa-1,3,5,7-tetramethyladamant-6-yl) proved to be much more suitable for facilitating the carbonylation reaction of the amide substrate (Table 1 , entries $3-8$ ). The catalysts supported by

Table 1. Pd-catalyzed cyclo-amidocarbonylation of PA: Screening of ligands. ${ }^{\text {[a] }}$

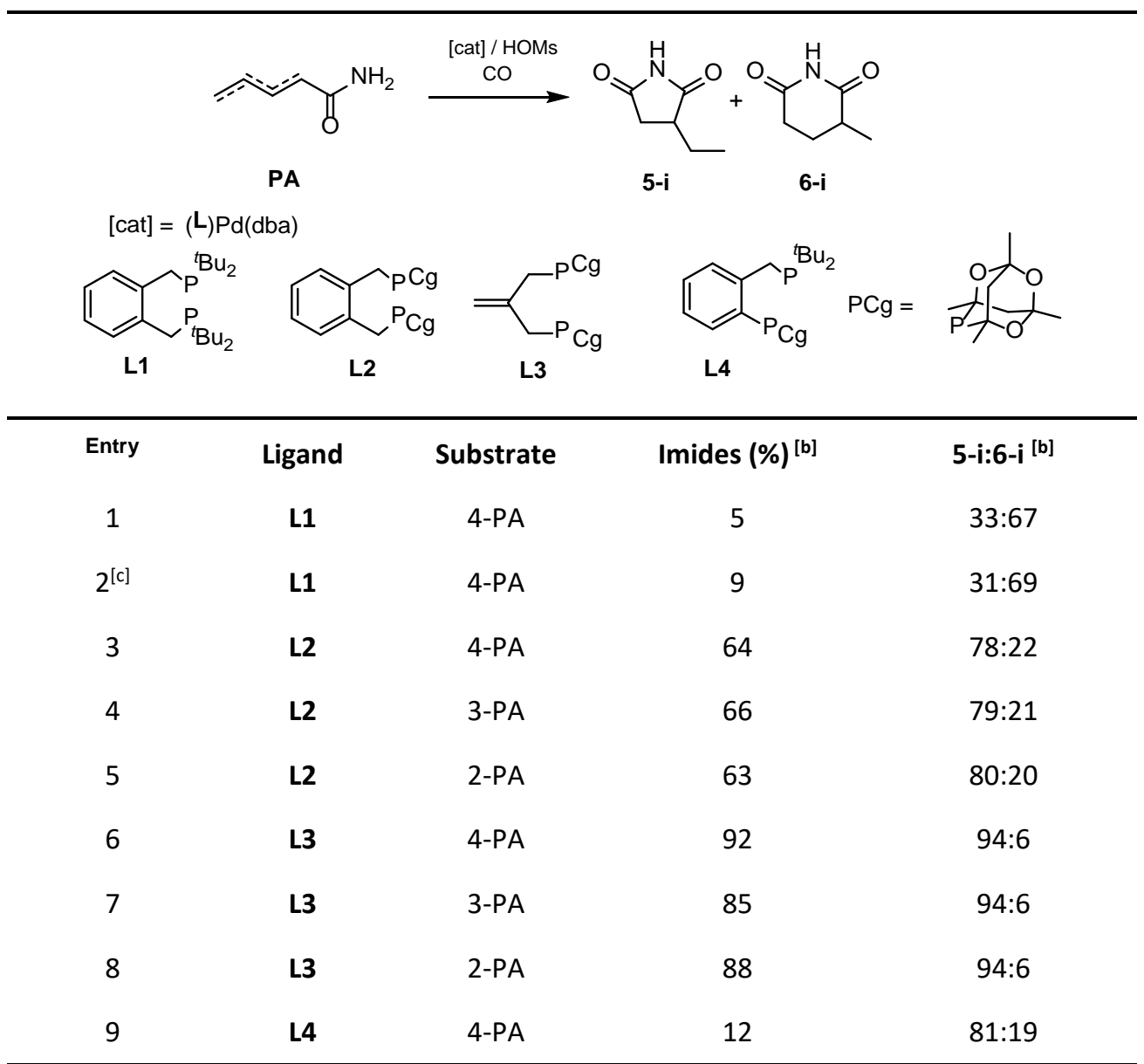

[a] Reaction conditions: $1 \mathrm{mmol}$ of PA, $0.5 \mathrm{~mol} \%$ [(L)Pd(dba)] (preformed), $15 \mathrm{~mol} \%$ $\mathrm{HOMs}, \mathrm{V}$ (diglyme) $=2.5 \mathrm{~mL}$, undecane (internal standard, $0.250 \mathrm{~mL}$ ), $100{ }^{\circ} \mathrm{C}, 16 \mathrm{~h}, \mathrm{P}_{\mathrm{CO}}=$ 50 bar. [b] Determined by GC using undecane as an internal standard. The yield of imides was in agreement with the conversion. [c] Same as [a] with catalyst prepared in situ from predissolved mixture of $\mathrm{Pd}(\mathrm{OAc})_{2}$ and $\mathbf{L}(1: 1.2)$ to which $15 \mathrm{~mol} \% \mathrm{HOMs}$ ( $v s$. PA) is added. 
ligands L2-L4 in combination with HOMs (30 eq/Pd) as co-catalyst, showed the exclusive formation of cyclic imide products $\mathbf{5}-\mathbf{i}$ and $\mathbf{6 - i}$. Remarkably, with both ligands $\mathbf{L} \mathbf{2}$ and $\mathbf{L} \mathbf{3}$ the yields and ratio of isomeric products obtained are independent of the starting PA isomer as substrate. The regioselectivity to 6-i appeared significantly higher for catalysts supported by L2 (Table 1, entries 3-5).

\section{Attempts to influence the regioselectivity results in different chemoselectivity}

We aimed to explore which parameters could influence the regioselectivity and examined a variety of conditions. The carbonylation reaction with 4-PA as substrate was conducted as a model reaction using $\mathbf{L} 2$ as ligand. As we anticipated that the interaction of the pendent amide functionality of the pentenamide substrate is important in the formation of the ring-closed product, we hoped to identify additives which could either interact with the Pd-bound pendent amide functionality or mimic its ability to reversibly coordinate to Pd and consequently influence the catalysts' regioselectivity. We focused on investigating the use of $\mathrm{N}$-methylpyrrolidone (NMP) as a compound that could likely compete with the coordination of the amide group and promote the isomerization to proceed to the terminal position. Surprisingly, instead of forming 7-i, the presence of NMP led to the formation of isomeric cyanoacids (CAs) 2-methyl-4-cyanobutyric acid (MCA) and 2-ethyl-3-cyanopropionic acid (ECA) (Table 2). ${ }^{[24]}$

Among a group of related additives \{dimethylformamide (DMF), dimethylacetamide (DMA), diethylacetamide (DEA), N-methylthiopyrrolidone (thio-NMP)\}, NMP turned out to be the most effective to promote cyanoacid formation at $100{ }^{\circ} \mathrm{C}$ (Table S7). Further experiments showed the use of lower temperatures to also favor cyanoacid formation. These experiments were done at higher PA concentration to compensate for the decrease in conversion caused by the presence of the promoter and the lower reaction temperature (Tables S8-9). In the absence of promoter, but at a reaction temperature of $60{ }^{\circ} \mathrm{C}$ the increase in substrate concentration already leads to the formation of the two cyanoacids ECA and MCA (5\%, Table 2, entry 1 and 2). This shows that the substrate amide functionality of PAs themselves to a certain extent can exert a similar effect as the addition of an external $\mathrm{N}$-alkylated amide. The addition of NMP has the most prominent effect when combined with the catalytic system using L2 (64\% selectivity to CAs vs. 36\% of imides when starting from 4-PA, entry 3), however at low conversions (11-17\%). The use of $\mathbf{L} 3$ gives better yields of cyclo-carbonylation products $(60 \%)$ and lower cyanoacid selectivity ( $30 \%$ CAs vs. $70 \%$ imides, entry 8). Similar to the experiments without NMP in Table 1, we also observed that in presence of NMP and even at the lower reaction temperature of $60{ }^{\circ} \mathrm{C}$ the catalytic regioselectivity was largely independent from the starting PA isomer substrate, both with $\mathbf{L} 2$ (Table 2, entries 3 and 4) and with L3 (Table 2, entries 8 and 9). Only with DMA added, it seemed that the catalyst regioselectivity became somewhat more dependent on the starting PA isomer (Table 2, entries 5 and 6 ). The separation of ECA and MCA on GC furthermore showed that similarly to the cycloimide products $\mathbf{5}-\mathbf{i}$ and $\mathbf{6}-\mathbf{i}$, the isomeric ratio ECA:MCA increases when using L3 (93\% ECA with L3 vs.62\% with L2, from 4-PA, see Table S9).

Using the catalyst and co-catalyst at half the concentration results in very similar selectivities (Table 2, entry 10 vs. entry 8). Applying a lower CO pressure of 5 bar instead of the common CO pressure of 50 bar (Table 2, entry 11) gives a significantly higher yield of $\mathbf{5}-\mathbf{i}$, but with a remarkable reduction in yield of CAs. A higher reaction temperature $\left(120^{\circ} \mathrm{C}\right.$, Table 2 , entry 12 , cf. entry 3 at $\left.60^{\circ} \mathrm{C}\right)$ also leads to increased yield of imides $5-i / 6-i$, with no observable formation of CAs.

Finally, exclusively imides were formed when using $N$-methyl-2-pentenamide as the substrate, predominantly the $\mathrm{N}$-methyl-2-ethylsuccinimide isomer (Tabel 2, entry 13). During the course of our study, Beller et al. reported Pd-catalyzed intermolecular amidocarbonylation reactions, using however exclusively $\mathrm{N}$-alkylated secondary amides and terminal alkenes as substrates under essentially acid-free conditions. ${ }^{[16]}$ We also applied the amidocarbonylation conditions of this report using $\mathrm{Pdl}_{2}$ as $\mathrm{Pd}$ source and oxydi-2,1-phenylene bis(diphenylphosphane) (DPEPhos) as ligand for our substrates 2-, 3-, or 4-PA, thus containing a primary amide functionality. The ring-closing carbonylation reaction only occurred when employing 4-PA, the substrate with a 
Table 2. Amidocarbonylation of pentenamides: the formation of cyanoacids MCA and ECA vs. imides. ${ }^{[a]}$

\begin{tabular}{|c|c|c|c|c|c|}
\hline & $\mathrm{H}_{2}$ & $5-i$ & -i & $\mathrm{ECA}$ & MCA \\
\hline Entry & Ligand & Substrate & Promoter & Yield $(\%)^{[b]}$ & 5-i:6-i:CAs ${ }^{[\mathrm{b}]}$ \\
\hline $1^{[\mathrm{c}]}$ & L2 & 4-PA & - & 51 & $65: 30: 5$ \\
\hline $2^{[c]}$ & L2 & 3-PA & - & 48 & $66: 29: 5$ \\
\hline 3 & L2 & 4-PA & NMP & 17 & $17: 19: 64$ \\
\hline 4 & L2 & 3-PA & NMP & 11 & $26: 34: 40$ \\
\hline 5 & L2 & 4-PA & DMA & 10 & $22: 24: 54$ \\
\hline 6 & L2 & 3-PA & DMA & 14 & $35: 30: 35$ \\
\hline 7 & L3 & 4-PA & - & 52 & $86: 14: 0$ \\
\hline 8 & L3 & 4-PA & NMP & 61 & $62: 8: 30$ \\
\hline 9 & L3 & 3-PA & NMP & 49 & $61: 7: 32$ \\
\hline $10^{\text {[d] }}$ & L3 & 4-PA & NMP & 53 & $61: 9: 30$ \\
\hline $11^{[\mathrm{e}]}$ & L3 & 4-PA & NMP & 84 & $87: 5: 8$ \\
\hline $12^{[f]}$ & L2 & 4-PA & NMP & 79 & $78: 22: 0$ \\
\hline $13^{[\mathrm{g}]}$ & L3 & N-Methyl-2-PA & NMP & N.D. ${ }^{[\mathrm{h}]}$ & $86: 14^{[i]}$ \\
\hline
\end{tabular}

[a] 2 mmol of PA, 1 mol\% (L)Pd(dba), 30 mol\% HOMs, V(promoter) = $1 \mathrm{~mL}$ (5 eq vs PA), undecane (IS: $0.250 \mathrm{~mL}$ ), $60{ }^{\circ} \mathrm{C}, 16 \mathrm{~h}, \mathrm{P}_{\mathrm{CO}}=50 \mathrm{~b}$. [b] Yield and selectivity in carbonylation products (imides + cyanoacids) were determined by GC using undecane internal standard. The sum of the yields of imides and CAs was in agreement with the conversion; CAs (cyanoacids) = MCA + ECA. [c] No promoter, V(diglyme) $=1 \mathrm{~mL}$. [d] Same as [a] but with $0.5 \mathrm{~mol} \%$ [(L)Pd(dba)], 15 mol\% HOMs. [e] same as [a] but Pco $=5$ bar. [f] Same as [a] but with $0.25 \mathrm{~mol} \%$ [(L)Pd(dba)], $7.5 \mathrm{~mol} \% \mathrm{HOMs}$, $\mathrm{V}($ diglyme+promoter $\{5$ eq. vs $\mathrm{PA}\})=2.5 \mathrm{~mL}$, undecane $(\mathrm{IS}): 0.250 \mathrm{~mL}, 120{ }^{\circ} \mathrm{C}, 16 \mathrm{~h}$, $\mathrm{P}_{\mathrm{CO}}=50$ bar. [g] $2 \mathrm{mmol} \mathrm{N}$-methyl-2-PA, 1 mol\% Pd(OAc)2, $1.2 \mathrm{~mol} \%$ L3, $10 \mathrm{~mol} \%$ HOMs, $V(N M P)=1 \mathrm{~mL}, 60{ }^{\circ} \mathrm{C}, 16 \mathrm{hr}$. Yield was not determined. [h] N.D. : exact yield not determined. [i] The products from this reaction are 5-membered ring $N$-methyl-2ethylsuccinimide and 6-membered ring $N$-methyl-2-methylglutarimide.

terminal alkene. We did not obtain any conversion using 3-PA or 2-PA as substrate, in agreement with the work of Beller, who reported that internal alkenes are totally unreactive under these conditions. The reaction using the Beller conditions with 4-PA as substrate yielded 6-i and the desired 7-i in very low yields $(1.5 \%$ and $1.2 \%$, respectively). Surprisingly, also the isomeric cyanoacids MCA and 5-cyanovaleric acid (CVA), and some compounds with higher GC retention time were formed, presumably intermolecular reaction products (dimers and oligomers; see Figure S1). The acid-free protocol reported by Beller et al. ${ }^{[16]}$ thus appeared to be unsuitable for the synthesis of 7-i from internal pentenamides. 


\section{NMR study}

It was surmised that the coordination mode of the primary amide functionality of the substrate at a cationic $[\mathrm{Pd} \text {-acyl }]^{+}$species might play a role in determining the chemoselectivity of amidocarbonylation. Therefore we undertook an NMR study to elucidate the possible effects of the strong acid co-catalyst and the basic selectivity modifier NMP on the coordination mode of amides to a Pd" centre. To this end, $\left[\mathrm{Pd}(\mathrm{MeCN})_{4}\right]\left(\mathrm{BF}_{4}\right)_{2}$ and 1.05 eq of ligand $\mathbf{L} \mathbf{3}$ were dissolved in deuterated acetone; to this solution acetamide was added, serving as a mimic for the amide functionality. Because ligand-exchange reactions at the Pd" ion occur at the NMR timescale, very broad signals were observed on ${ }^{1} \mathrm{H}$ NMR for the $\mathrm{NH}_{2}$ protons of acetamide at room temperature. The solution was cooled down to $-65^{\circ} \mathrm{C}$ in order to slow down the ligand-exchange kinetics (see Figure S2). A rather complex spectrum was obtained due to different coordination modes and the inequivalence of the two $\mathrm{NH}_{2}$ protons. To differentiate the tautomeric forms of the amide (two protons on the $\mathrm{N}$ ) from the iminol (one proton on the $\mathrm{N}$, one proton on the O), ${ }^{15} \mathrm{~N}$-labeled acetamide was used. Every proton attached directly to the ${ }^{15} \mathrm{~N}$ of acetamide is split into two peaks with a large ${ }^{1} J_{H-N}(\sim 90 \mathrm{~Hz})$ coupling, and therefore ${ }^{15} \mathrm{~N}$-decoupled proton NMR spectra were recorded to simplify the spectra (Figure 1, bottom trace). By means of a ${ }^{1} \mathrm{H}-{ }^{15} \mathrm{~N}$ HSQC measurement (Figure S3), three differently coordinated acetamide species were identified, all having two inequivalent protons bound to the nitrogen atom. The ${ }^{15} \mathrm{~N}$ chemical shifts were found at $124.4,126.3$ and $130.4 \mathrm{ppm}$ tentatively assigned to species A, B and C (Figure 1). We did not observe the iminol tautomer, that was previously shown to be the favored coordination mode for acetamide to a [(diethylenetriamine) $\left.\mathrm{Pt}^{\prime \prime}\right]^{2+}$ centre. ${ }^{[25]}$ For two of the observed species ( $\mathbf{A}$ and $\mathbf{B}$ ), the $\mathrm{N}-\mathrm{H}$ peaks in ${ }^{1} \mathrm{H}$ NMR were in rapid exchange with free acetamide (indicated with ${ }^{*}$ ), as observed by positive cross-peaks in NOESY (Figure S7). The remaining species C was not exchanging rapidly with free acetamide, indicating that compound $\mathbf{C}$ is kinetically more stable. It has been reported that the kinetic product of amide coordination to $\mathrm{Pt}$ " is bound with the $\mathrm{O}$ atom, and that the thermodynamically more stable $\mathrm{N}$ coordination requires heating or a longer period of reaction time. ${ }^{[25-27]}$ Therefore, in analogy, we tentatively assign species $\mathbf{A}$ and $\mathbf{B}$ as two isomers coordinated to $\mathrm{Pd}^{\prime \prime}$ with the $\mathrm{O}$ of acetamide, and species $\mathbf{C}$ as an $\mathrm{N}$ coordinated isomer (see Scheme 2). ${ }^{[25-27]}$

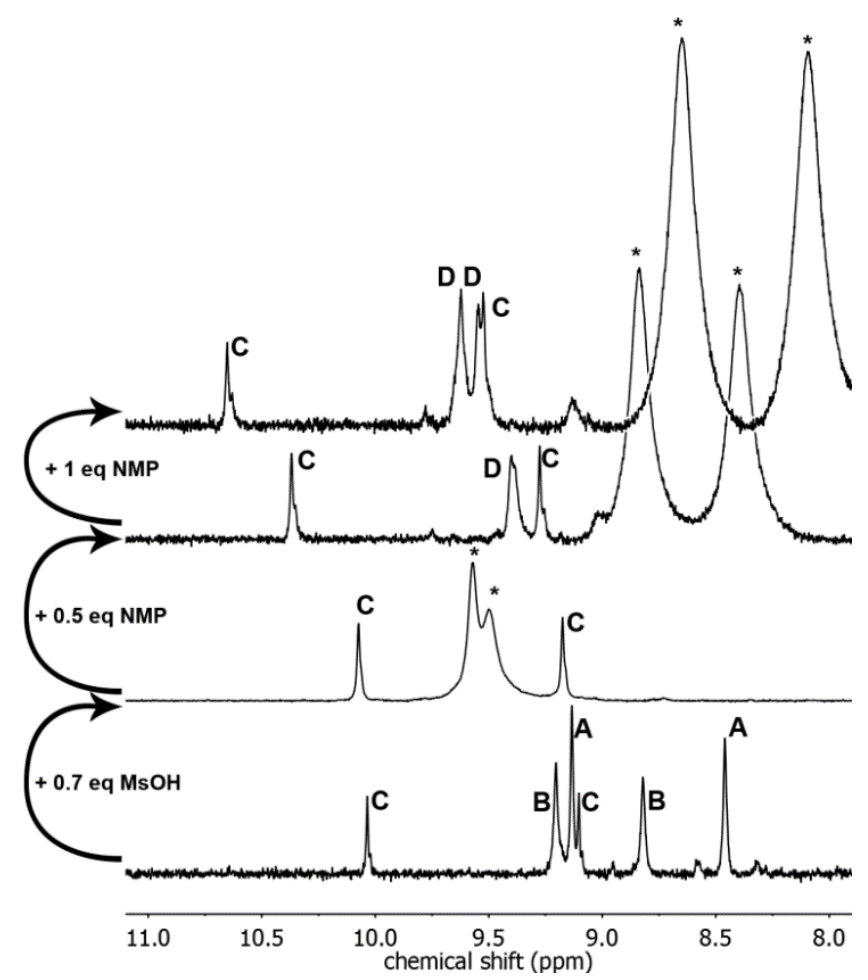

Figure 1. ${ }^{1} \mathrm{H}\left\{{ }^{15} \mathrm{~N}\right\} \mathrm{NMR}$ spectra of a solution of $\left[\mathrm{Pd}(\mathrm{MeCN})_{4}\right]\left(\mathrm{BF}_{4}\right)_{2}(20 \mu \mathrm{mol}), \mathbf{L} 3(21 \mu \mathrm{mol})$ and ${ }^{15} \mathrm{~N}$-acetamide $(68 \mu \mathrm{mol})$ in acetone- $\mathrm{d}_{6}(0.6 \mathrm{~mL})$. The $\mathrm{NH}_{2}$ protons of different coordinated acetamide species $(\mathbf{A}, \mathbf{B}, \mathbf{C}$ and $\mathbf{D})$, as well as free acetamide $\left({ }^{*}\right)$ are indicated. The spectra with added HOMs (0.7 eq to Pd), and HOMs + NMP (0.5 eq to Pd, and 1.5 eq to Pd in total) are also shown. 


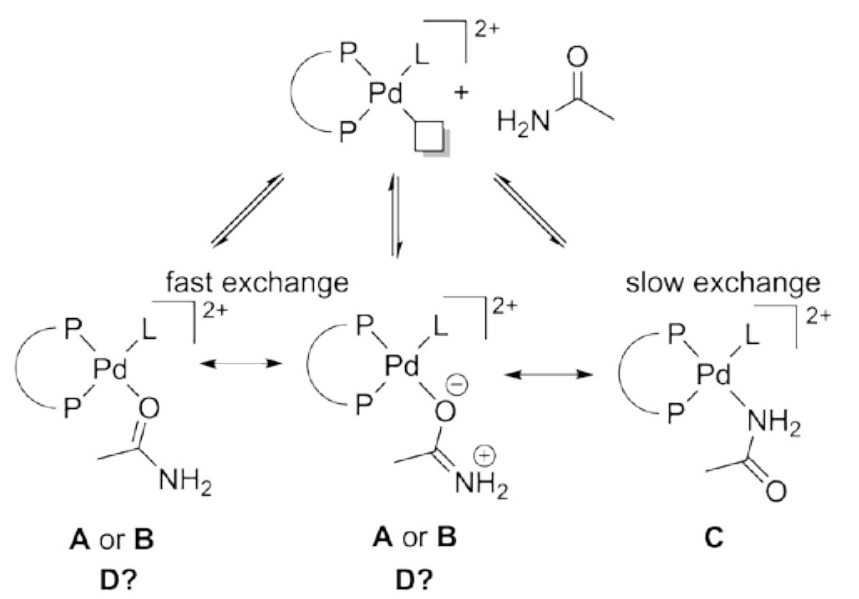

Scheme 2. Proposed coordination modes of acetamide to $[\mathrm{Pd}(\mathrm{L} 3)(\mathrm{L})]^{2+}(\mathrm{L}=\mathrm{MeCN}$, acetone, or $\mathrm{NMP})$.

Upon the addition of HOMs ( $2 \mu \mathrm{L}, 0.7$ eq to $\mathbf{P d}$ ), species $\mathbf{A}$ and $\mathbf{B}$ disappear, indicating dissociation of the amide (the peaks collapsed into two broad signals, connected to a single ${ }^{15} \mathrm{~N}$ at $125.8 \mathrm{ppm}$ of free acetamide, ${ }^{*}$ ), as shown in Figure 1. The peaks of species $\mathbf{C}$ shifted but remained. The only mode of coordination for acetamide observed in the presence of HOMs thus is the $\mathrm{N}$-coordinated species $\mathbf{C}$. The reason that upon addition of acid the amide dissociates from species $\mathbf{A}$ and $\mathbf{B}$ can be understood as a competition between the binding of a soft Lewis-acid palladium ion or the hard Lewis-acid proton to the hard Lewis-base $\mathrm{O}$ atom. In contrast, $\mathrm{N}$ protonation is highly unfavored, ${ }^{[28]}$ therefore such competition is absent for species $\mathbf{C}$.

Upon addition of NMP the strength of the acid is reduced by almost an order of magnitude (pKa of HOMs $=-1.9$, while pKa of protonated $\mathrm{N}$-methylacetamide $=-1),{ }^{[28]}$ leading to an upfield shift of the free acetamide peaks $(*)$ and appearance of another type of coordinated acetamide (species $\mathbf{D}$ ). At 0.5 equivalent of NMP a broad peak is observed in proton NMR that splits into two peaks upon addition of more NMP ( $30 \mu \mathrm{L}, 1.5$ eq to Pd in total). The chemical shift of the ${ }^{15} \mathrm{~N}$ of this species is at $126.7 \mathrm{ppm}$, which corresponds roughly to that for species $\mathbf{B}$ in the absence of HOMs or added NMP, and we therefore conclude a similar coordination mode to be present in species D (Figures S2-4). The observation of a single broad peak in the presence of a small amount of NMP might be due to fast proton exchange at the amide $\mathrm{N}$ atom, leading to an averaged signal. We assigned coordination mode B as an O-coordinated acetamide species; it thus seems reasonable to conclude that the presence of a large amount of the weak base NMP leads to the reappearance of an O-coordinated amide Pd" species.

\section{Intermolecular amidocarbonylation}

To connect our studies with the intermolecular amidocarbonylation of 1-octene with $\mathrm{N}$-methylacetamide under acid-free conditions recently reported by Beller et al., ${ }^{[16]}$ we repeated one of the reported experiments (i.e., 2 mmol 1-octene, $2 \mathrm{mmol} N$-methylacetamide, 0.5 mol\% Pdl 2,1 mol\% DPEPhos, $2 \mathrm{~mL}$ diglyme, $\mathrm{T}=105{ }^{\circ} \mathrm{C}, \mathrm{P}_{\mathrm{co}}=50$ bar). As reported, the corresponding $N$-methylimide was produced (Table 3 , entry 1 ). 
Table 3. Chemoselectivity towards imide or carboxylic acid/nitrile formation for different substrates and conditions in the intermolecular amidocarbonylation reaction.

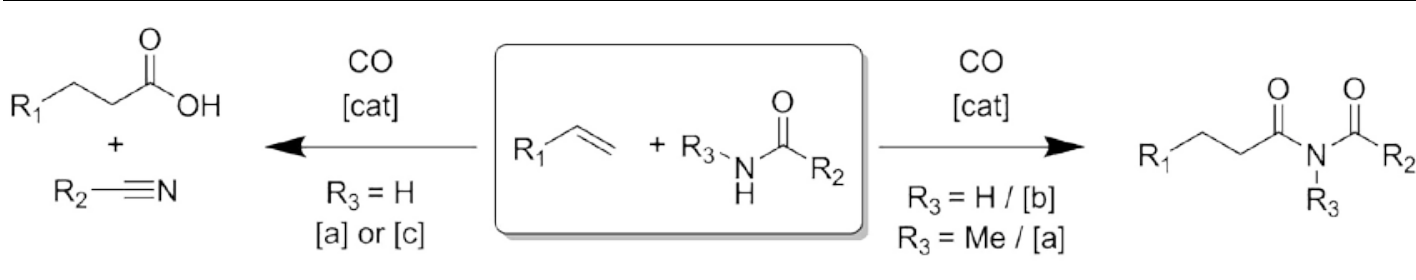

\begin{tabular}{|c|c|c|c|}
\hline entry & substrates & conditions & Main product \\
\hline 1 & $\begin{array}{c}\text { 1-octene } \\
N \text {-methyl acetamide }\end{array}$ & [a] & $\mathrm{N}$-methyl- $\mathrm{N}$-acetyInonanamide \\
\hline 2 & $\begin{array}{l}\text { 1-octene } \\
\text { acetamide }\end{array}$ & [a] & $\begin{array}{l}\text { nonanoic acid } \\
\text { acetonitrile }^{[d]}\end{array}$ \\
\hline 3 & $\begin{array}{c}\text { 1-octene } \\
\text { pentanamide }\end{array}$ & [a] & $\begin{array}{l}\text { nonanoic acid } \\
\text { pentanenitrile }\end{array}$ \\
\hline 4 & $\begin{array}{c}\text { 1-octene } \\
\text { pentanamide }\end{array}$ & [b] & $N$-pentanoylnonanamide \\
\hline 5 & $\begin{array}{c}\text { 1-octene } \\
\text { pentanamide }\end{array}$ & [c] & $\begin{array}{l}\text { nonanoic acid } \\
\text { pentanenitrile }\end{array}$ \\
\hline
\end{tabular}

[a] $2 \mathrm{mmol}$ 1-octene, $2 \mathrm{mmol}$ amide, $0.5 \mathrm{~mol} \% \mathrm{Pdl}_{2}, 1 \mathrm{~mol} \%$ DPEPhos, $\mathrm{V}$ (diglyme) $=2 \mathrm{~mL}, 105{ }^{\circ} \mathrm{C}, 20$ h, Pco $=50$ bar. [b] $2 \mathrm{mmol} 1$-octene, $2 \mathrm{mmol}$ pentanamide, $1 \mathrm{~mol} \% \mathrm{Pd}(\mathrm{OAc})_{2}, 1.2 \mathrm{~mol} \% \mathrm{~L} 3,10 \mathrm{~mol} \%$ HOMs, V(diglyme) $=2 \mathrm{~mL}, 105{ }^{\circ} \mathrm{C}, 16 \mathrm{~h}$. [c] 2 mmol 1-octene, $2 \mathrm{mmol}$ pentanamide, $1 \mathrm{~mol} \%$ $\mathrm{Pd}(\mathrm{OAc})_{2}, 1.2 \mathrm{~mol} \% \mathrm{~L} 3,10 \mathrm{~mol} \% \mathrm{HOMs}, \mathrm{V}(\mathrm{NMP})=1 \mathrm{~mL}, 60{ }^{\circ} \mathrm{C}, 16 \mathrm{~h}$. [d] acetonitrile could not be observed quantitatively due to its low boiling point, overlapping with impurities on GC and its low response factor.

However, when non-alkylated acetamide was used instead, the almost exclusively observed products were nonanoic acid and a small quantity of 2-methyloctanoic acid, along with acetonitrile (Table 3, entry 2). When pentanamide was used instead of acetamide, pentanenitrile was quantitatively observed as product besides the carboxylic acids in a 1:1 ratio. In contrast, in the acidic conditions described in this work (Table 3, entry 4), the reaction between 1-octene and pentanamide indeed yielded the imide product alongside small amounts of nonanoic acid and 2-methyloctanoic acid. Furthermore, when the reaction was performed in the presence of acid, but in NMP at $60^{\circ} \mathrm{C}$ (Table 3, entry 5), again only nonanoic acid, 2-methyloctanoic acid and pentanenitrile were formed, similarly to the intramolecular formation of cyanoacids ECA and MCA when PA is used as the substrate.

\section{Discussion}

\section{Catalysis of isomerizing cyclo-amidocarbonylation of PA}

Above we present a new Pd-catalyzed carbonylative transformation of pentenamides - termed isomerizing cyclo-amidocarbonylation - to afford cyclic imides in high yields. The transformation is composed of a tandem sequence of reactions, i.e., alkene bond isomerization and intramolecular amidocarbonylation. Although the exact mechanism of the reactions must be subject of further study, our catalytic results suggest that the reaction proceeds via a similar mechanistic pathway as the well-studied isomerizing alkoxy- or hydroxycarbonylation of alkenes with [ $\mathrm{LPd}-\mathrm{H}]^{+}$as the actual active catalyst species. ${ }^{[17-19,29-30]}$

The first Pd-H species can generally be formed in situ from a variety of palladium sources. In our reaction, the active $\mathrm{Pd}^{\prime \prime}-\mathrm{H}$ catalyst is generated in situ by reacting a strong acid (e.g. HOMs) with either a preformed $\mathrm{LPd}^{0}$ 
complex or with a 1:1 mixture of a Pd" salt with a ligand $\mathrm{L}$, in the presence of $\mathrm{CO}$ and PA as substrate. The use of a preformed $\mathrm{LPd}^{0}$ precursor proved to be a most convenient and reproducible protocol for the formation of an active catalytic system (Tables 1,2$)$. This is in line with the general observation that $\left[\operatorname{L1Pd}^{0}(\mathrm{dba})\right]$ in combination with a strong acid serves as a suitable catalyst precursor mixture, by protonation of the $\mathrm{Pd}^{0}$ center generating the cationic species [ $\left.\mathbf{L 1 P d}^{\prime \prime}-\mathrm{H}\right]^{+}(\mathrm{OMs})^{-}$active in e.g. alkene methoxycarbonylation. ${ }^{[31]}$ Starting from a 1:1 mixture of a Pd" salt and a ligand $\mathbf{L}$ with excess of HOMs essentially the same catalyst complex is formed in situ. Catalyst formation supposedly proceeds under reaction conditions via stoichiometric carbonylative reduction of LPd" by the alkene and a nucleophilic reagent, such as water, alcohol (or in our case the amide). ${ }^{[30,32]}$ We have indeed experimentally verified that both protocols gave similar catalytic results (see Table 1, entries 1 and 2).

The species $\left[\mathrm{LPd}^{\prime \prime}-\mathrm{H}\right]^{+}(\mathrm{OMs})^{-}$can start the catalytic cycle for the reaction by migration of the hydride to a coordinated alkene; subsequent $\beta$-hydrogen elimination, migration and $\mathrm{CO}$ insertion generates a mixture of several $[\text { LPd-alkyl }]^{+}$and $[\text {LPd-acyl }]^{+}$species. It is thought that the final imide product-forming step involves a nucleophilic attack of the amide at the Pd-acyl species resulting in amidocarbonylation in analogy to the reaction with alcohols in alkoxycarbonylation or with water in hydroxycarbonylation. ${ }^{[19]}$ Although the detailed mechanism of this final step is not known, it is thought that the nucleophilic attack of the amide, in analogy with those of alcohols and water, ${ }^{[33-34]}$ involves $\mathrm{N}$-coordination of the amide at the [ $\mathrm{LPd}^{\mathrm{I}}$-acyl] $]^{+}$center followed by deprotonation of the coordinated amide, upon which the product imide is generated by reductive elimination. This reaction sequence thus formally generates an $\left[\mathrm{LPd}^{0}\right]$ species and one equivalent of $\mathrm{H}^{+}$, which together subsequently or concertedly regenerates [ $\left[\mathrm{LPd}^{\prime \prime}-\mathrm{H}\right]^{+}(\mathrm{OMS})^{-}$.

Reported mechanistic studies on related intermolecular isomerizing methoxycarbonylation reactions of alkenes catalyzed by cationic $[\mathrm{LPd}-\mathrm{H}]^{+}$complexes indicate relatively low barriers and therefore apparent reversibility of both migration/ $\beta$-elimination of the hydride as well as subsequent $\mathrm{CO}$ (de-) insertion steps in the Pd-alkyl species under the prevailing reaction conditions. ${ }^{[31]}$ The excess of a strong acid in our catalytic experiments fulfills a co-catalytic role, circumventing the buildup of $\mathrm{Pd}^{0}$ species through rapid protonation of any [ $\mathrm{LPd}^{0}$ ] species formed, thus avoiding regeneration of the actual active catalyst $\left[\mathrm{LPd}^{\prime \prime}-\mathrm{H}\right]^{+}(\mathrm{OMs})^{-}$to become rate determining (see Scheme 3). Furthermore, the excess of strong acid co-catalyst leads to rapid alkene bond isomerization by maintaining a relatively high concentration of $\left[\mathrm{LPd}^{\prime \prime}-\mathrm{H}\right]^{+}$. The general catalytic cycle proposed for isomerizing amidocarbonylation rationalizes the formation of cyclic imides such as 5-i, 6-i from PA as substrate (Scheme 3, two left reaction pathways).

\section{Regioselectivity in isomerizing cyclo-amidocarbonylation of PA}

Formation of 5-i was always favored when a catalytic system comprising one of the 'cage' ligands L2, L3 or L4 was used, with a 5-i:6-i regioselectivity of respectively 80:20, 94:6 and 81:19 (Table 1, entries 3, 6 and 9). Furthermore, it appeared that for catalysts supported by each of these ligands, not only the regioselectivity, but also the catalytic activity is independent of the double bond position in the starting PA isomer (Table 1, entries 3-5 for L2, and 6-8 for L3). These results thus show that one of the requirements for a selective isomerizing carbonylation reaction is fulfilled: alkene bond isomerization is much faster than the overall product formation rate. Moreover, the reaction selectively yielded imides only; no other products were observed. However, carbonylation and product formation clearly does not occur at the least hindered terminal C-atom (Scheme 3, right pathway to 7-i).

Based on the general mechanistic work on related intermolecular isomerizing carbonylation reactions, ${ }^{[29-30]}$ we can propose a likely rationalization of the observed regioselectivity. Although the catalyst has a high alkene bond isomerization activity, as deduced from the independence of the results on the used PA isomer, still only the 5-and 6-membered cyclic imides are produced. This outcome likely stems from the relative stabilities of an equilibrated mixture of the 5-, 6-membered and 7-membered palladacyclic species (comprising both the [LPd- 


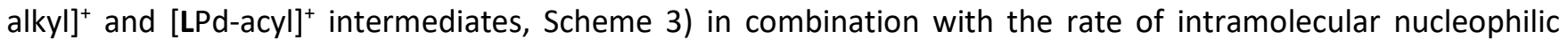
attack of the amide at the respective Pd-acyl species.

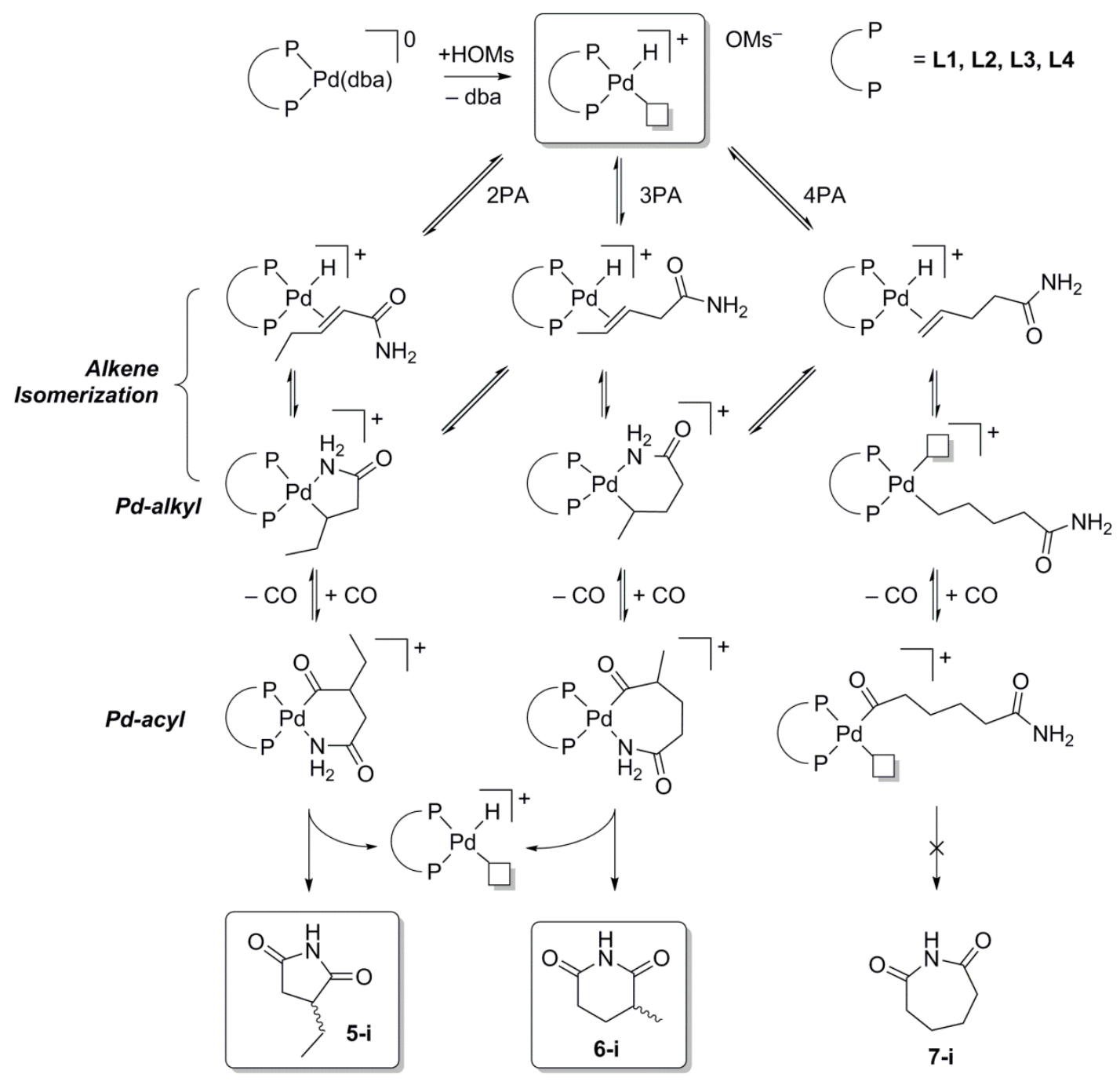

Scheme 3. Chelation control of the regioselectivity outcome in LPd-catalyzed PA cyclo-amidocarbonylation reaction. For clarity, weakly coordinated $\mathrm{OMs}^{-}$anions associated with catalytic [LPd] ${ }^{+}$centre not shown. The chelation of the amide in the rightmost pathway is depicted as absent to illustrate why 7-i is not formed.

One important kinetic factor of cyclic imide formation must be the local concentration of the pendent amide moiety in the neighborhood of the Pd center, which is determined by its chelation strength. We thus hypothetically identify chelation of the amide moiety as the main obstacle for amidocarbonylation at the least hindered terminal $\mathrm{C}$-atom of PA, since this would involve significantly less abundantly present 7-membered ring (Pd-alkyl) and 8-membered ring (Pd-acyl) intermediates in which chelation is weak (depicted as absent in Scheme 3, right pathway). In contrast to the strongly favored route towards 5-i involving a relatively stable 6membered ring Pd-acyl, the one yielding 6-i involves a less abundantly present 7-membered ring Pd-acyl complex. Comparing the results of the use of two similar cage ligands $\mathbf{L} \mathbf{2}$ and $\mathbf{L} \mathbf{3}$, it is clear that $\mathbf{L} \mathbf{2}$ brings about more steric congestion at the coordination sites opposite the phosphorus donors due to a larger bite-angle of the longer C4 spacer, which destabilizes chelation and results in lower overall rates of total imide (5-i+6-i) formation as is apparent from Table 1 (entries 2-4 for $\mathbf{L} \mathbf{2}$ and 5-7 for L3). At the same time, the higher steric congestion induced by $\mathbf{L} \mathbf{2}$ destabilizes the ethyl-substituted Pd-alkyl complex, instead driving the complex to form more of the methyl-substituted Pd-alkyl intermediate rationalizing the lower $\mathbf{5 - i : 6 - i ~ r a t i o ~ f o r ~} \mathbf{L} 2$ relative to L3. A plausible explanation for the poor performance of $\mathbf{L} \mathbf{1}$ in our case would then be that its induced steric congestion by the bulkier $-\mathrm{P}\left({ }^{t} \mathrm{Bu}\right)_{2}$ moieties at the $\mathrm{Pd}$ center is such that it prevents chelation to take place, thereby hampering the ring-closing termination reaction. However, electronic factors operating both in 
chelation of the [LPd-acyl] ${ }^{+}$species and the resulting nucleophilicity of the amide cannot be excluded either, as the cage ligands $\mathbf{L} \mathbf{2}$ and $\mathbf{L} \mathbf{3}$ bear electron-withdrawing tris-oxa-adamantyl substituents $v s$. the electron-donating tert-butyl substituents in L1. The electron-withdrawing ligands could favor coordination of the amide and thereby the succeptibility of the [LPd-acyl] ${ }^{+}$intermediate for nucleophilic attack by rendering the Pd center more electrophilic.

\section{Chemoselectivity in (cyclo)amidocarbonylation}

Our NMR study indicates that both $\mathrm{N}$ - and O-coordinated amide Pd" species are present in an LPd(II)/acetamide mixture in neutral conditions. The change in chemoselectivity of $\mathrm{LPd}^{\prime \prime} / \mathrm{H}^{+}$catalyzed amidocarbonylation of PA from imides to cyanoacids with addition of NMP correlates well with our NMR study indicating exclusive $\mathrm{N}$-coordination of amide to a LPd" species to persist under acidic conditions. Upon addition of the weakly basic NMP, and thus when acidity is quenched, the NMR signals belonging to compound ' $D$ ' indicate re-appearance of an O-coordinated $\mathrm{Pd}^{\prime \prime}$ amide species. We thus explain the observed different chemoselectivity of amidocarbonylation in absence or presence of acid with the mechanism depicted in Scheme 4 . The amide coordination behavior studied with a cationic $\mathrm{LPdX}_{2}\left(\mathrm{X}=\mathrm{BF}_{4}{ }^{-}\right)$species can be related to coordination of the pendent amide moiety of $\mathrm{PA}$ in catalytically relevant cationic [ $\mathrm{LPd}-\mathrm{acyl}]^{+}\left(\mathrm{OMs}^{-}\right)$species. In view of the similarity in cationic nature of both Pd centers, we consider such an extrapolation to be a reasonable assumption. Thus as shown in Scheme 4, N-coordination of the amide moiety (PdN) to an LPd"-acyl species leads to the formation of imides via nucleophilic attack of this intermediate (reaction $\mathbf{c}$ ), whereas O-coordination existing under acidquenching conditions (PdO) could hypothetically lead to isoimides via nuclophilic attack of the carbonyl $\mathrm{O}$ atom (reaction d). The cyanoacid could be formed by a subsequent rearrangement of the isoimide via reaction $\mathbf{e}$. Isoimides are indeed quite unstable molecules; ${ }^{[35-36]}$ they have been suggested to be intermediates in the formation of the thermodynamically favored imides (reaction $\mathbf{f}$ ). ${ }^{[35-36]}$

As isoimides were not observed in any product mixture an immediate in situ rearrangement to isomeric imides and/or cyanocarboxylic acid should thus be considered. DFT energy calculations on the three isomeric compounds 2-ethyl-succinimide (5-i), 2-ethyl-succinisoimide (5-ii) and ECA showed that the isoimide (5-ii) is thermodynamically the most unstable compound of the three. The $\Delta G$ values (gas phase) of 5-ii and ECA relative to 5 -i are +16.9 and $+14.2 \mathrm{kcal} \cdot \mathrm{mol}^{-1}$ (Figure 2). The same trend was found for the regioisomers $6-\mathrm{i}, 6-\mathrm{ii}$, and MCA, as well as for $\mathbf{7 - i}, 7-\mathbf{i i}$, and CVA; the isoimides 6 -ii and 7 -ii are thermodynamically less favored by +22.8 and $+19.2 \mathrm{kcal} \cdot \mathrm{mol}^{-1}$ compared to their imide isomers $6-\mathrm{i}$ and $7-\mathrm{i}$, respectively (see ESI Table S10). Furthermore, the transition state calculated for the uncatalyzed rearrangement reaction $\mathbf{f}$ for cyclic isoimides to imides (i.e., from 5-ii to 5-i) is very high in energy $\left(\sim+57.4 \mathrm{kcal} \cdot \mathrm{mol}^{-1}\right.$, see ESI Table S10), making such rearrangement highly unlikely to occur. The rearrangement to cyanoacid (reaction e) might be feasible as such a rearrangement involves a likely lower activation barrier for hydrogen transfer from $\mathrm{N}$ to $\mathrm{O}$ with simultaneous $\mathrm{O}-\mathrm{CN}$ bond rupture. Unfortunately, our attempts in finding an accessible transition state using DFT energy calculations remained elusive. Therefore, this pathway to cyanoacids as yet can neither be proven nor excluded on theoretical grounds. However, in all three cases the cyanoacids are thermodynamically favored over the isoimides.

As a diagnostic tool we conducted an amidocarbonylation experiment with $\mathrm{N}$-methyl-2-PA as substrate (Table 2, entry 13) under conditions favorable for formation of cyanoacids with PA. As rearrangement from an $N$-methyl isoimide to cyanoacid is impossible, it should be possible to identify $\mathrm{N}$-methyl-2-ethylisoimide, if formed in a reaction mixture, by NMR and/or GLC analysis. Rearrangement to the cyclic $N$-methyl imide can also be excluded as such a rearrangement would likely have to surmount a similarly high activation barrier as 5-ii (>50 $\mathrm{kcal} / \mathrm{mol}$ ). However, in the actual experiment $\mathrm{N}$-methyl-2-ethylsuccinimide (and not the corresponding isoimide) was observed as the exclusive product of cyclo-amidocarbonylation, giving a strong indication that the isoimides are neither formed as intermediate products to cyanoacids in the amidocarbonylation of PAs. This 
thus suggests that the envisaged intermediate $\mathrm{Pd}$-iminolate in species $\mathbf{P d O}$ does not undergo iminolysis of $\mathrm{Pd}$ acyl (Scheme 4, reaction d) at a sufficiently high rate under the applied mild conditions $\left(60^{\circ} \mathrm{C}\right.$ ), as this step apparently involves a too high energy barrier. The reaction system will then rather proceed via the existing equilibrium with $\mathbf{N}$-coordinated species $\mathbf{P d N}$, and subsequent nucleophilic attack involving a significantly lower energy barrier to produce exclusively the thermodynamically most stable imide $\mathbf{5}-\mathbf{i}$. As indicated by the DFT calculated energy differences between isoimides and imides, it can be indeed reasonably assumed that the activation energy for iminolyis in species $\mathbf{P d O}$ as rate-determining step resulting in a $\sim 15 \mathrm{kcal} / \mathrm{mol}$ higher-energy isoimide product will be significantly higher than the activation energy for rate-determining imidolysis in species PdN forming the thermodynamically most stable imide product (Bell-Evans-Polanyi principle). ${ }^{[37]}$

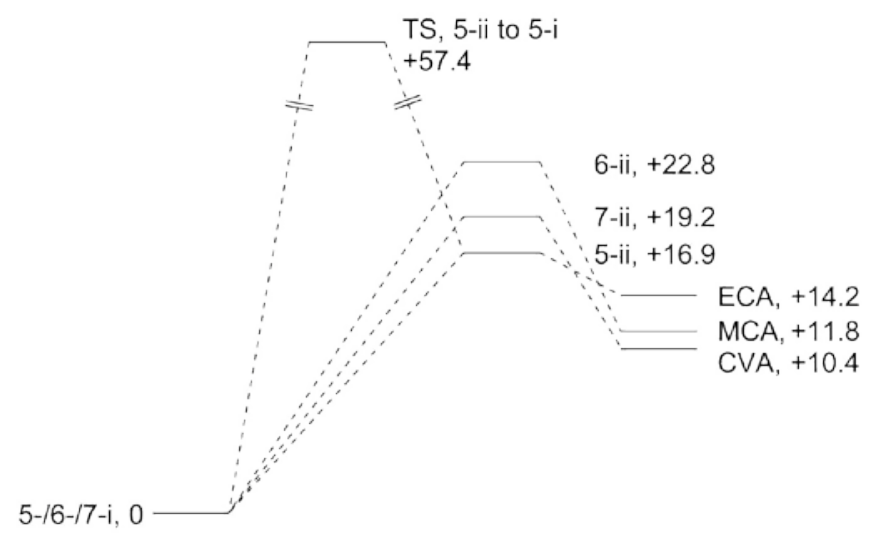

Figure 2. Schematic representation of the energies (in $\mathrm{kcal} / \mathrm{mol}$ ) calculated for the various (potential) carbonylation products of pentenamide.

On the other hand, the absence of isoimide formation with $N$-methyl-2-PA is also a clear signpost that an alternative low barrier route to cyanoacids must exist in amidocarbonylation of PAs, which does not involve isoimides as intermediates. We therefore envisage a direct pathway to cyanoacids via reactions $\mathbf{g}$ and $\mathbf{h}$ in Scheme 4. This pathway can be viewed as an intramolecular dehydrating hydroxycarbonylation of PAs. Dehydration of the amide moiety leads to Pd-acyl bound water, a direct intermediate in subsequent hydroxycarbonylation of the alkene moiety of PAs. There is precedent of Pd"-catalyzed dehydration of primary amides and for $\mathrm{H}_{2} \mathrm{O}$ transfer between primary amides and nitriles. ${ }^{[38]}$ Dehydration of the amide moiety likely involves a sequence of $\mathrm{H}$ - and $\mathrm{OH}$ transfers from the iminol moiety to the $\mathrm{Pd}$ center, but a concerted transfer to $\mathrm{Pd}$-acyl thus reforming $\mathrm{Pd}-\mathrm{H}$ together with the carboxylic and nitrile moieties could also be envisaged. The exact mechanism for dehydrating hydroxycarbonylation of PAs is not known, but it seems reasonable to assume that the hydrolysis step of the intermediate Pd-acyl species provides a lower energy barrier than the preceding dehydration of the amide moiety, thereby avoiding water build-up. The alternative two-step process, i.e. dehydration of PAs and subsequent intermolecular hydroxycarbonylation of pentenenitrile as separate steps, is less likely to occur because of two reasons. First, in a control experiment the hydroxycarbonylation of pentenenitrile/ $\mathrm{H}_{2} \mathrm{O}$ mixtures could not be demonstrated with the catalyst systems under the prevailing acidic catalytic conditions in this work (see ESI). Instead, only traces of imides (5-i, 6-i) and CVA were formed due to rapid deactivation of the catalytic system by consumption of the co-catalyst HOMs via stoichiometric hydrolysis of pentenenitrile (producing pentenoic acid and the salt $\mathrm{NH}_{4} \mathrm{OMs}$ which precipitated from solution), see ESI Table S1. Second, a build-up of $\mathrm{H}_{2} \mathrm{O}$ would thus lead to equimolar amounts of pentenenitriles, which were not observed. Indeed, when the amount of acid was increased to a 1:1 stoichiometry with respect to the substrate the only product that was observed was adipic acid, i.e., through full hydrolysis and hydroxycarbonylation of the PA substrate.

In another control experiment (see ESI) 3-cyanopropionic acid (ECA) was exposed to the L3Pd/HOMs catalyst system under a $\mathrm{CO}$ pressure (50 bar) at $105{ }^{\circ} \mathrm{C}$, which resulted in complete conversion to the corresponding 
imide (5i-i) in $16 \mathrm{~h}$, clearly indicating a $\mathrm{Pd}$-catalyzed reverse process from that of Scheme 4 . Interestingly, in the absence of $\mathrm{Pd}$ and $\mathrm{CO}$, but in the presence of $10 \mathrm{~mol} \% \mathrm{HOMs}$ only about $10 \%$ imide was formed in $16 \mathrm{~h}$. Although conducted at a higher temperature than the forward reactions reported in Table $2\left(60{ }^{\circ} \mathrm{C}\right)$, the result of this experiment suggests that the reaction pathway represented in Scheme 4 reactions $\mathbf{b}, \mathbf{g}$ and $\mathbf{h}$ likely provides a reversible pathway from CAs to the thermodynamically more stable imide with a sufficient rate at a higher reaction temperature. It thus suggests that ECA is a kinetic product under the low temperature conditions, which consecutively can be converted to the thermodynamically most stable imide 5-i. Thus, when amidocarbonylation is conducted at higher temperatures, even in the presence of NMP, CAs are not produced as final carbonylation products (Table 2, entry 12), i.e., consecutive conversion to the more stable imides prevails under these conditions. The decreased formation of CA's at low CO pressures, in particular in favour of 5-i (Table 2, entries 8, 11), could be a reflection of the influence of $\mathrm{CO}$ binding on the coordination mode of the pendent amide moiety. Thus less $\mathrm{CO}$ present seems to favour $\mathrm{N}$-amide coordination, possibly because a high concentration of $\mathrm{CO}$ prevents the rearrangement from the O-coordinated amide to the thermodynamic $\mathrm{N}$ coordinated product.

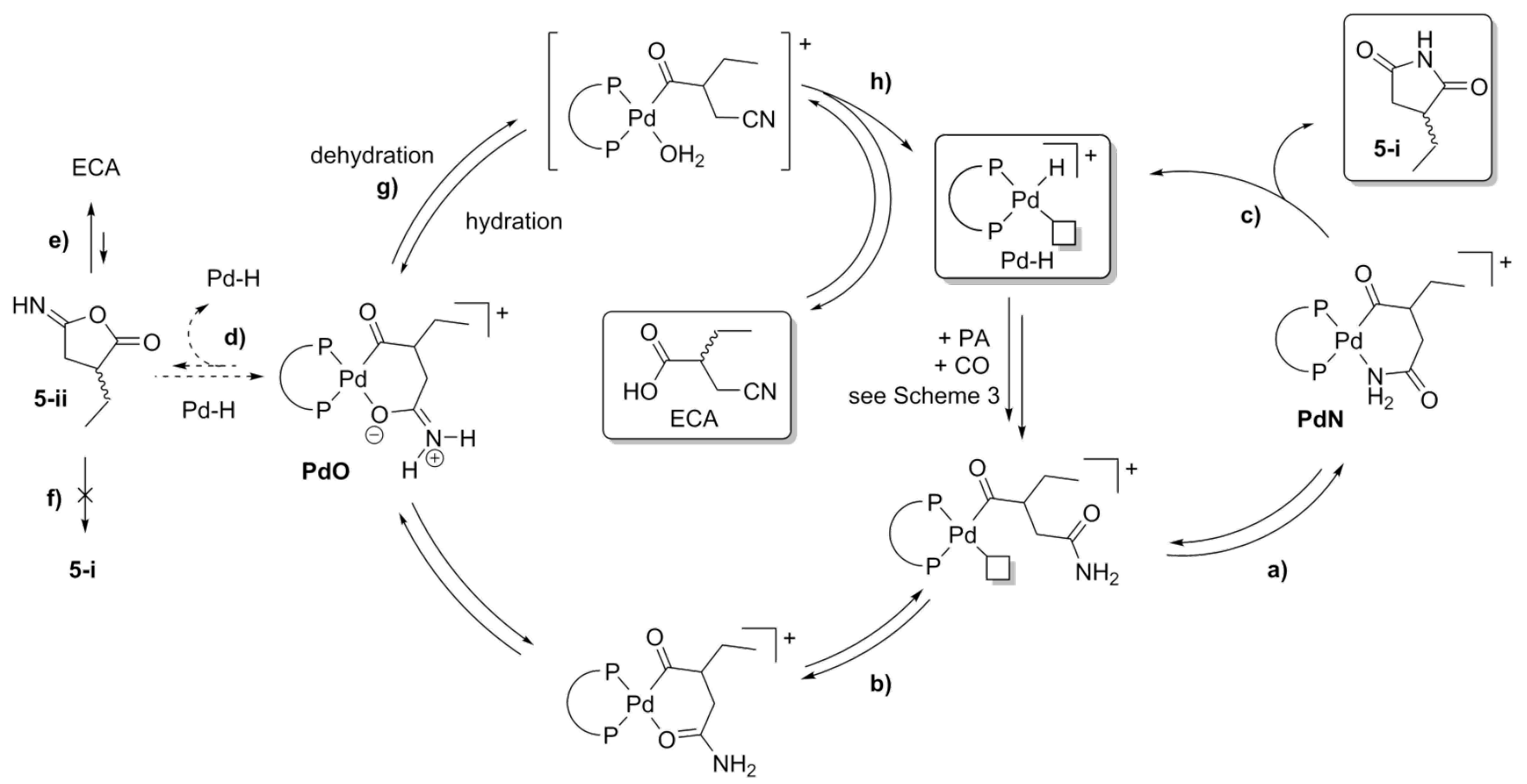

Scheme 4. Reaction scheme explaining the chemoselectivity for the amidocarbonylation of pentenamides; all palladium species in this scheme are Pd". Displayed here: regioselectivity towards 5-membered Pd-alkyl ring forming ethyl-branched products (see Scheme 3); weakly coordinated anions $\left(\mathrm{OMs}^{-}\right.$) associated with catalytic [LPd] ${ }^{+}$centre not shown. a) In the presence of strong acid in diglyme, the amide group coordinates through the $\mathrm{N}$ atom $(\mathbf{P d N})$. b) In the absence of strong acid, or when the acid is quenched by basic solvents such as NMP, the amide can coordinate through the $\mathrm{O}$ atom (PdO). $\mathrm{C}$ ) 'Ring-closing elimination' leads to direct formation of the imide product $\mathbf{5 - i}$, and (P-P)Pd', which is rapidly or concertedly protonated to reform the active catalyst Pd-H. d) 'Ring-closing elimination' may lead to formation of the isoimide 5-ii (an unstable intermediate), and ( $\mathrm{P}-\mathrm{P}) \mathrm{Pd}^{0}$, which is rapidly or concertedly protonated to reform the active catalyst $\mathrm{Pd}-\mathrm{H}$. e) The unstable intermediate 5-ii rearranges to give the cyanoacid product ECA. f) The rearrangement to indirectly form 5-i is unlikely because of a large activation barrier due to ring strain. g) Alternative direct pathway to ECA: the O-coordinated amide can be dehydrated which subsequently leads to $h$ ) concerted hydroxycarbonylation of the intermediate cyanoacyl$\mathrm{Pd}-\mathrm{OH}_{2}$ species.

Consequences for intermolecular amidocarbonylation. Finally, we relate the experimental findings of intermolecular amidocarbonylation (Table 3) with the mechanistic model we developed in Scheme 4 for the intramolecular amidocarbonylation of PAs. Clearly the characteristics of inter- and intramolecular amidocarbonylation must be highly similar. Notably, under acidic conditions the $\mathrm{N}$-coordinated $\mathrm{Pd}$-amide exists and the catalytic events will lead to imides as the products (Table 3, entry 4). These products are a result of 
nucleophilic attack of the amide substrate of the open chain Pd-C8-acyl. Under quenched-acid conditions (or so called acid-free conditions in the Beller protocol $\left.{ }^{[16]}\right)$, similar to the intramolecular version of amidocarbonylation the existence of O-coordinated Pd amide precursors can lead to coproduction of a C9 carboxylic acid together with the nitrile derived from the applied amide substrate (Table 3 entries 2, 3 and 5). Such a conversion is likely to occur via dehydrating hydroxycarbonylation, like the intramolecular version producing cyanoacids from PAs. However, when using $\mathrm{N}$-methyl acetamide as the nucleophile a mechanism involving an $\mathrm{H}$-transfer process in the dehydrating process at the Pd center obviously cannot take place. Therefore, under acid-free conditions, the hypothetical and thermodynamically unfavorable $N$-methyl-nonanoylmethylisoimide should be formed by the iminolysis reaction of the Pd-acyl iminolate. However, this $\mathrm{N}$-methyl isoimide was not observed; exclusively $\mathrm{N}$-acetyl- $\mathrm{N}$-methylnonanamide was formed (Table 3, entry 1 ). This means that equilibria between $\mathrm{O}$ - and $\mathrm{N}$ coordinated amide-Pd species (analogous to those indicated in Scheme 4) cause a shift in product formation via the $\mathrm{N}$-coordinated species, thus ultimately exclusively forming the imide product.

\section{Conclusion}

We show that Pd-catalyzed intramolecular amidocarbonylation of pentenamides can lead to two types of isomeric carbonylation products. In the presence of strong acid (pKa $<<$ ), 5 - and 6-membered cyclic imides are formed in high selectivity. However, in the absence of strong acid, or when the strong acid is quenched by addition of an external amide such as NMP, cyanocarboxylic acids are produced. An NMR spectroscopic study showed the involvement of several possible modes of amide coordination to a cationic (P-P)Pd" center, depending on the presence or absence of acid. In combination with a catalytic study also involving intermolecular amidocarbonylation using several primary and secondary amides as substrates it was deduced that the dependence of the catalytic results on the $\mathrm{pH}$ has its origin in the prevailing coordination mode of the (chelating) amide functionality. In the presence of strong acid imides must be formed via $\mathrm{N}$-coordination of the amide to Pd". Under neutral conditions, however, the kinetically favored O-coordination of the (chelating) amide leads to the formation cyanocarboxylic acids. It is proposed that these cyanoacids are formed as kinetic products by a novel type of Pd catalyzed conversion termed 'dehydrating hydroxycarbonylation' of PAs in which the primary amide functionality is dehydrated at $\mathrm{Pd}$ while the formed $\mathrm{H}_{2} \mathrm{O}$ is concertedly consumed in hydroxycarbonylation of the PAs alkene moiety. Under elevated temperatures the cyanoacids can be converted to the thermodynamically more stable imides via the reversed Pd-catalyzed pathway. It is shown that intermolecular amidocarbonylation applied to separate alkenes and amide nucleophilic reagents as substrates, proceeds via an analogous mechanistic pathway. With primary amides and under acid-free or acid-quenched reaction conditions amidocarbonylation of an alkene produces selectively carboxylic acids and nitriles in a 1:1 ratio with high rates. Under acidic conditions, however, $\mathrm{N}$-acyl amides can also be selectively produced from the same substrates. Experiments with $\mathrm{N}$-alkylated amides as substrate have been diagnostic in developing plausible mechanistic schemes of Pd-catalyzed cyclo-amidocarbonylation of (functional) alkenes under both acidic and acid-free conditions.

In the presence of a strong acid as a co-catalyst double-bond isomerization appears to be much faster than ultimate product formation, therefore opening up the possibility to selectively produce linear imides from internal alkenes and both primary and secondary amides as the nucleophiles. In the case of ring-closing amidocarbonylation starting from pentenamides, however, the chelation strength of the amide functionality to palladium determines which product is formed. Because the chelation strength becomes smaller with increasing size of the palladacycle, only 5-i and a small amount of 6-i were obtained. In conclusion, we have overcome two of the challenges of the bio-based synthesis of 7-i: chemoselectivity to form imides and fast double-bond isomerization. The last challenge is to overcome the regioselectivity induced by the chelation of the pendent amide. To prevent this unfavorable chelation is subject of further study. 


\section{Experimental}

General. A glovebox of M. Braun Inert gas-System GmbH, Germany, was used for storing and handling of airsensitive phosphane ligands. All reaction preparations and manipulations were performed using standard Schlenk techniques under an argon atmosphere (i.e. catalytic procedure, collecting, drying, degassing solvents). The stainless steel autoclave reactors $(100 \mathrm{~mL})$ were of HEL Limited, UK, equipped with magnetic stirrer, pressure transducer and temperature controlling thermocouple. A Hewlett Packard HP6890 Series auto-sampler GC system was used for regular GC analysis (FID detector), together with a column from Agilent (HP-1MS UI; 30 m, $0.25 \mathrm{~mm}$ i.d., $1 \mu \mathrm{m}$ film thickness) using helium as carrier gas. The separation of imides $\mathbf{5 - i}, \mathbf{6}-\mathbf{i}$, and $\mathbf{7}$-i as well as cyanoacids 5-cyanovaleric acid (CVA), 2-methyl-4-cyanobutyric acid (MCA) and 2-ethyl-3-cyanopropionic acid (ECA), was performed on a Varian CP.3800 GC, with an Alltech column (AT ${ }^{\mathrm{TM}}$-WAX; $30 \mathrm{~m}, 0.53 \mathrm{~mm}, 2 \mu \mathrm{m}$ film thickness), using helium as carrier gas. GC-MS analysis were carried out on an Agilent technologies 7820A GC system series coupled with an Agilent technologies 5975 series GC-MSD system, and a column from Agilent (DM-5MS UI, $30 \mathrm{~m}, 0.25 \mathrm{~mm}, 1 \mu \mathrm{m}$ film thickness), using helium as carrier gas. GC-yields were calculated using undecane as internal standard. The approximation was made that $\mathbf{5 - i ,} \mathbf{7 - i}$, and the corresponding cyanoacids, since they show the same empirical formula, had the same response factor as 6 -i with the FID detector. Nuclear magnetic resonance spectra were recorded on a Bruker DPX300 $(300 \mathrm{MHz})$ spectrometer, on a Bruker AV-400 $(400 \mathrm{MHz}$ ) spectrometer, or on a Bruker AV-500 (500 MHz) spectrometer. Chemical shifts (ppm) are given relative to solvent or to TMS. High resolution mass spectra (HRMS) were recorded on LTQ-orbitrap mass spectrometer (Thermo Fisher Scientific, Waltham, USA). The data are given as mass units per charge $(\mathrm{m} / \mathrm{z})$. For preformed $\operatorname{Pd}(\mathbf{L})(\mathrm{dba})$ and imides the ionization technique used was is ESI (Electro Spray lonization). HRMS of the cyanoacids were also performed with APCI (Atmospheric Pressure Chemical lonization). Purification of the products was conducted with technical grade solvents and silica gel 60, 40-63 $\mu \mathrm{m}$ (Screening Devices bv).

Gases. Carbon monoxide and dihydrogen were purchased from Linde.

Chemicals. $\mathrm{Pd}(\mathrm{dba})_{2}$ and $\mathrm{Pd}(\mathrm{OAc})_{2}$, were purchased from Sigma-Aldrich. $\mathrm{Pd}(\mathrm{TFA})_{2}$ and $\mathbf{L 1}$ (dtbpx) were purchased from Strem. The cage ligands L2, L3, L4 were donated by Shell (where they were previously synthesized according to literature procedures). ${ }^{[39]}$ Pentenoic acids (trans 2-pentenoic acid, 3-pentenoic acid, 4pentenoic acid), used for the synthesis of pentenamide substrates (PAs), were purchased from Sigma Aldrich (2and 3-PA) and Alfa Aesar (4-PA). Undecane (internal standard) was purchased from Merck. All other reagents (acids, promoters) were purchased from Sigma-Aldrich. The liquids were degassed and dried over molecular sieves, and stored together with the solids in a glovebox under argon. 2-Methyl glutarimide (6-i) was purchased from Novochemy as a pure authentic sample, which allowed a GC calibration vs. undecane in GC. Adipimide (7-i) was purchased from Uorsy.

Substrates. 3-PA, 4-PA were synthesized as reported. ${ }^{[8-9]}$ The same procedure was utilized to produce 2-PA (from trans-2-pentenoic acid). After synthesis of each pentenamide the obtained white solid was dissolved in a minimum amount of dichloromethane and reprecipitated with cold hexane $\left(-20^{\circ} \mathrm{C}\right)$. The crystalline solid was then filtered, washed with cold hexane, and dried in vacuo. The resulting white powder was ultimately filtered over a pad of basic alumina (dichloromethane was used as eluent) to provide us with analytically pure pentenamides.

\section{Typical batch autoclave reaction with the catalytic system comprising $\mathrm{Pd}(\mathrm{L})(\mathrm{dba})+30$ eq HOMs}

The substrates 2-, 3-, or 4-PA (99.1 mg, $1 \mathrm{mmol}$ ) were weighed in a glass insert (containing an oven-dried stirring bar) which was subsequently introduced into an autoclave reactor. The autoclave was tightly closed and subsequently filled with argon, using a Schlenk line connected to one of the valves of the autoclave. In parallel, $\mathrm{Pd}(\mathrm{L1})$ (dba) $(4.4 \mathrm{mg}, 5 \mu \mathrm{mol})$ was dissolved in dried and degassed diglyme $(2.5 \mathrm{~mL}$ ) in a Schlenk flask (in a glovebox under argon). Methane sulfonic acid $(9.8 \mu \mathrm{L}, 0.15 \mathrm{mmol})$ was added to this yellow solution, which 
turned immediately red. Undecane $(1.185 \mathrm{mmol}, 0.250 \mathrm{~mL})$ was then added as an internal standard and the solution was eventually transferred to the autoclave via cannula (through another valve, under a continuous flow of argon). After 5 minutes at RT the autoclave was flushed with $\mathrm{CO}$, and then pressurized with 50 bar of $\mathrm{CO}$. The reaction mixture was heated to $100{ }^{\circ} \mathrm{C}$ (within about $30 \mathrm{~min}$ ) under stirring at $500 \mathrm{rpm}$. All reaction conditions of the catalytic process were controlled by computerized software panels. After standing for 16 hours at this temperature, the autoclave was cooled down to room temperature over approximately one hour and subsequently vented to atmospheric pressure. Alterations to this procedure are explained in the ESI.

\section{Acknowledgements}

This research was performed within the framework of the CatchBio program. We gratefully acknowledge the support of the SmartMix Program of the Netherlands Ministry of Economic Affairs and the Netherlands Ministry of Education, Culture and Science. Royal Dutch Shell plc is kindly acknowledged for a generous donation of phosphane ligands. We are grateful to Gerwin K. Spijksma for mass spectrometry measurements, Fons Lefeber and Dr. Karthick B. Sai Sankar Gupta for their help with the NMR studies and to Jos van Brussel for GC expertise.

\section{Supporting information}

Supporting information is available: synthetic procedures of the complexes, synthesis and characterization of the products and catalytic procedures are supplied. Tables describing optimization of product formation are supplied (Table S2-S9). NMR spectra of the coordination of acetamide to L3Pd" are supplied (Figures S2-S7). Computational details on the DFT calculations of products and intermediates are supplied (Tables S10-13).

\section{References}

[1] A. Corma, S. Iborra, A. Velty, Chem. Rev. 2007, 107, 2411-2502.

[2] J.-P. Lange, R. Price, P. M. Ayoub, J. Louis, L. Petrus, L. Clarke, H. Gosselink, Angew. Chem. Int. Ed. 2010, 49, 4479-4483.

[3] R.-J. van Putten, J. C. van der Waal, E. de Jong, C. B. Rasrendra, H. J. Heeres, J. G. de Vries, Chem. Rev. 2013, 113, 1499-1597.

[4] J. J. Bozell, L. Moens, D. C. Elliott, Y. Wang, G. G. Neuenscwander, S. W. Fitzpatrick, R. J. Bilski, J. L. Jarnefeld, Resour. Conserv. Recy. 2000, 28, 227-239.

[5] W. R. H. Wright, R. Palkovits, ChemSusChem 2012, 5, 1657-1667.

[6] J. M. Thomas, R. Raja, Proc. Natl. Acad. Sci. USA 2005, 102, 13732-13736.

[7] D. Li, F. Shi, S. Guo, Y. Deng, Tetrahedron Lett. 2005, 46, 671-674.

[8] S. Raoufmoghaddam, M. T. M. Rood, F. K. W. Buijze, E. Drent, E. Bouwman, ChemSusChem 2014, 7, 1984-1990.

[9] S. Raoufmoghaddam, E. Drent, E. Bouwman (Leiden University), WO2014163506, 2014.

[10] X. Fang, R. Jackstell, M. Beller, Angew. Chem. Int. Ed. 2013, 52, 14089-14093.

[11] X. Fang, H. Li, R. Jackstell, M. Beller, J. Am. Chem. Soc. 2014, 136, 16039-16043.

[12] C. Jimenez-Rodriguez, A. A. Nunez-Magro, T. Seidensticker, G. R. Eastham, M. R. L. Furst, D. J. ColeHamilton, Catal. Sci. Tech. 2014, 4, 2332-2339.

[13] B. El Ali, K. Okuro, G. Vasapollo, H. Alper, J. Am. Chem. Soc. 1996, 118, 4264-4270.

[14] H. Liu, N. Yan, P. J. Dyson, Chem. Commun. 2014, 50, 7848-7851.

[15] J. Liu, H. Li, A. Spannenberg, R. Franke, R. Jackstell, M. Beller, Angew. Chem. Int. Ed. 2016, 55, 1354413548.

[16] H. Li, K. Dong, H. Neumann, M. Beller, Angew. Chem. Int. Ed. 2015, 54, 10239-10243.

[17] E. Drent, W. Jager (Shell), WO2001068583, 2001.

[18] C. Jimenez Rodriguez, D. F. Foster, G. R. Eastham, D. J. Cole-Hamilton, Chem. Commun. 2004, 1720-1721.

[19] C. H. Low, J. D. Nobbs, M. van Meurs, L. P. Stubbs, E. Drent, S. Aitipamula, M. H. L. Pung,

Organometallics 2015, 34, 4281-4292. 
[20] J. Falbe, F. Korte, Angew. Chem. Int. Ed. Eng. 1962, 1, 266-267.

[21] J. Falbe, F. Korte, Chem. Ber. 1962, 95, 2680-2687.

[22] R. Aoun, J.-L. Renaud, P. H. Dixneuf, C. Bruneau, Angew. Chem. Int. Ed. 2005, 44, 2021-2023.

[23] A. M. Smith, R. Whyman, Chem. Rev. 2014, 114, 5477-5510.

[24] M. Ptaszek, D. Lahaye, M. Krayer, C. Muthiah, J. S. Lindsey, J. Org. Chem. 2010, 75, 1659-1673.

[25] T. C. Woon, D. P. Fairlie, Inorg. Chem. 1992, 31, 4069-4074.

[26] T. C. Woon, W. A. Wickramasinghe, D. P. Fairlie, Inorg. Chem. 1993, 32, 2190-2194.

[27] D. P. Fairlie, T. C. Woon, W. A. Wickramasinghe, A. C. Willis, Inorg. Chem. 1994, 33, 6425-6428.

[28] H. Sigel, R. B. Martin, Chem. Rev. 1982, 82, 385-426.

[29] P. Roesle, L. Caporaso, M. Schnitte, V. Goldbach, L. Cavallo, S. Mecking, J. Am. Chem. Soc. 2014, 136, 16871-16881.

[30] P. Roesle, C. J. Dürr, H. M. Möller, L. Cavallo, L. Caporaso, S. Mecking, J. Am. Chem. Soc. 2012, 134, 17696-17703.

[31] W. Clegg, M. R. J. Elsegood, G. R. Eastham, R. P. Tooze, X. Lan Wang, K. Whiston, Chem. Commun. 1999, 1877-1878.

[32] A. Marson, A. B. van Oort, Wilhelmus P. Mul, Eur. J. Inorg. Chem. 2002, 2002, 3028-3031.

[33] J. K. Liu, B. T. Heaton, J. A. Iggo, R. Whyman, Chem. Commun. 2004, 1326-1327.

[34] P.W.N.M. van Leeuwen, M. A. Zuideveld, B. H. G. Swennenhuis, Z. Freixa, P. C. J. Kamer, K. Goubitz, J. Fraanje, M. Lutz, A. L. Spek, J. Am. Chem. Soc. 2003, 125, 5523-5539.

[35] C. K. Sauers, J. Org. Chem. 1969, 34, 2275-2279.

[36] J. S. P. Schwarz, J. Org. Chem. 1972, 37, 2906-2908.

[37] K. D. S. Bromberg, Molecular Driving Forces, Garland Science, New York, 2003.

[38] S. I. Maffioli, E. Marzorati, A. Marazzi, Org. Lett. 2005, 7, 5237-5239.

[39] T. Fanjul, G. Eastham, M. F. Haddow, A. Hamilton, P. G. Pringle, A. G. Orpen, T. P. W. Turner, M. Waugh, Catal. Sci. Tech. 2012, 2, 937-950.

\section{Table of Contents}

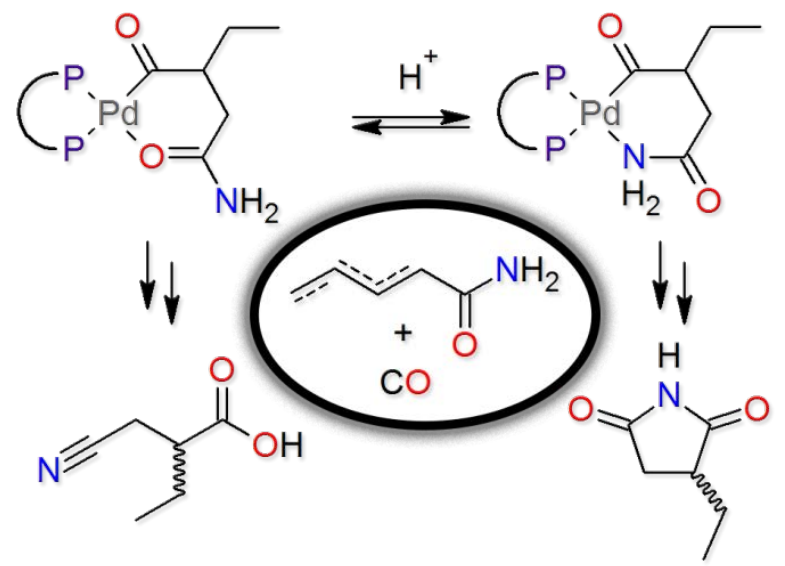

A new isomerizing ring-closing amidocarbonylation reaction is reported. From an NMR study it was deduced that $\mathrm{N}$-coordination of the amide moiety is responsible for a high selectivity to cyclic imide products, whereas O-coordination of the amide functionality leads to the formation of cyanoacids. 